COLO-HEP-340, LPTHE-Orsay-94-62

\title{
Ising Spins on Thin Graphs
}

\author{
C.F. Baillie \\ Dept of Computer Science, University of Colorado \\ Boulder, CO 80309, USA \\ D.A. Johnston ${ }^{1}$ and J. P. Kownacki \\ LPTHE \\ Universite Paris Sud, Batiment 211 \\ F-91405 Orsay, France
}

28 July 1994

\begin{abstract}
The Ising model on "thin" graphs (standard Feynman diagrams) displays several interesting properties. For ferromagnetic couplings there is a mean field phase transition at the corresponding Bethe lattice transition point. For antiferromagnetic couplings the replica trick gives some evidence for a spin glass phase. In this paper we investigate both the ferromagnetic and antiferromagnetic models with the aid of simulations. We confirm the Bethe lattice values of the critical points for the ferromagnetic model on $\phi^{3}$ and $\phi^{4}$ graphs and examine the putative spin glass phase in the antiferromagnetic model by looking at the overlap between replicas in a quenched ensemble of graphs. We also compare the Ising results with those for higher state Potts models and Ising models on "fat" graphs, such as those used in $2 \mathrm{D}$ gravity simulations.
\end{abstract}

Submitted to Nucl. Phys. B. [FS]

1 Address: Sept. 1993 - 1994, Permanent Address: Maths Dept, Heriot-Watt University, Edinburgh, Scotland 


\section{Introduction}

There has been a considerable amount of both analytical and numerical work on the Ising model living on dynamically triangulated random surfaces and the dual $\phi^{3}$ (and also $\phi^{4}$ ) graphs in the past few years, largely motivated by the interest of the model for string theory and two-dimensional gravity. The partition function of the model for a fixed number of points $n$ is

$$
Z_{n}(\beta)=\sum_{G^{n}} \sum_{\{\sigma\}} \exp \left(\beta \sum_{<i j>} G_{i j}^{n} \sigma_{i} \sigma_{j}\right)
$$

where $G_{i j}^{n}$ is the connectivity matrix for a given graph or triangulation and the sum over graphs $\sum_{G^{n}}$ means that we are, in effect, looking at annealed connectivity disorder in such models. The model was solved in the planar limit in [i] by noting that a grand canonical partition function could be obtained as the free energy of a zero dimensional $N \times N$ hermitian matrix model [2]

$$
F=\frac{1}{N^{2}} \log \int D^{N^{2}} \phi_{a} D^{N^{2}} \phi_{b} \exp \left(-\frac{1}{2} \phi_{a} K_{a b} \phi_{b}+V\left(\phi_{a}, \phi_{b}\right)\right)
$$

in the limit $N \rightarrow \infty$. The matrix propagator in the above is given by

$$
K_{a b}^{-1}=\left(\begin{array}{cc}
\sqrt{g} & \frac{1}{\sqrt{g}} \\
\frac{1}{\sqrt{g}} & \sqrt{g}
\end{array}\right)
$$

where $g=\exp (2 \beta)$, and the potentials for $\phi^{3}$ and $\phi^{4}$ graphs are given by

$$
V\left(\phi_{a}, \phi_{b}\right)=\frac{\lambda}{3}\left(\phi_{a}^{3}+\phi_{b}^{3}\right)
$$

and

$$
V\left(\phi_{a}, \phi_{b}\right)=\frac{\lambda}{4}\left(\phi_{a}^{4}+\phi_{b}^{4}\right)
$$

respectively. We have suppressed the external field dependence for clarity, though unlike the case of a fixed 2d lattice an exact solution is still possible even in the presence of an external field. The diagrams generated by the matrix model integral are "fat" graphs because the two indices carried by the matrix fatten the propagators into ribbons. This gives enough structure to build surfaces with the trace round internal loops and the initial factors in the coupling and propagator producing just the right factor of $N$ to count the genus. The matrix model solution has been pushed as far as toroidal topology [3], and both the toroidal and planar results for the (non-Onsager) critical exponents are in agreement with continuum derivations in lightcone and conformal gauge Liouville theory [- 4 . There is now a gratifyingly large body of numerical evidence supporting the dynamical lattice exponents for both Ising and Potts models [5].

Divorcing the Ising model calculations and simulations from their origins in string theory and regarding them as an exercise in statistical mechanics, it is natural to ask if the methods employed might also be applicable to quenched disorder. In such a case the free energy $F$ is calculated on each graph before summing over the random graphs

$$
F=\sum_{G} \log Z(G)
$$

where $Z(G)$ is the partition function for the Ising model on a particular random graph. The logarithm can be dealt with by using the replica trick [6]

$$
\begin{aligned}
\log Z & \simeq \frac{Z^{n}-1}{n} \\
n \rightarrow 0 &
\end{aligned}
$$

at the expense of having to calculate with $n$ replicas of the original matter system. On a fixed lattice this is not a problem, but the $c=1$ barrier in $2 \mathrm{~d}$ gravity means that it isn't possible to calculate with more 
than 2 Ising replicas. Ignoring the problem and simply taking the limit $n \rightarrow 0$ in the formulae of [4 for the critical exponents, does however give apparently reasonable results that are close to the dynamical lattice exponents, which is what is seen in a direct simulation of a quenched set of $\phi^{3}$ fat graphs [7].

It would obviously be better to have a more trustworthy formalism for investigating quenched problems where such troubles did not arise and one could confidently calculate with an arbitrary number of replicas. Such a formalism is actually inherent in the matrix model approach in a limit that is opposite in a sense to that usually considered, namely $N \rightarrow 1$ [8]. In this limit the fat graphs that build the surfaces or their duals in the matrix model integrals degenerate to "thin" graphs - in other words zero dimensional Feynman diagrams. Such graphs have in fact been considered before in the context of spin glass theories [9], without utilizing the methods of field theory and large order expansions of [8]. The generic problems in the matrix model approach for $c>1$ can be traced to the difficulty in evaluating angular integrals that arise from diagonalizing the matrices, and these disappear for $N=1$. Consequently the difficulties in applying the replica formalism also vanish. As the regular surface interpretation is lost $^{1}$ the continuum limit is taken by forcing the number of points $n \rightarrow \infty$ rather than tuning the cosmological constant as for $N \rightarrow \infty$.

In the remainder of the paper we discuss the behaviour of the Ising model on such thin graphs. In the next section we review the analytical calculations of [8] for ferromagnetic couplings, which serve as a test of the method. In this context we also discuss the saddle point solution for higher $\phi^{n}$ interactions and $q=3,4$ state Potts models and their close correspondance with Bethe lattice results. We then describe in section 3 simulations of the ferromagnetic Ising model on $\phi^{3}$ and $\phi^{4}$ graphs, carried out essentially as a test of the formalism and the simulation programs, which are in agreement with these results. In section 4 we discuss the anti-ferromagnetic Ising model on thin graphs and describe our simulations of both the Ising and three and four state Potts models with purely negative couplings on quenched ensembles of Feynman diagrams. The calculations of [8] suggest that the antiferromagnetic model may have a spin glass phase at sufficiently negative coupling, and we investigate this numerically by histogramming the overlaps between replicas. In sections 5 and 6 we discuss briefly some exploratory simulations of the Ising antiferromagnet on quenched fat graphs and of Potts antiferromagnets on thin graphs respectively, largely to compare the results with those for the Ising model. Finally in section 7 we present our conclusions and discuss the various loose ends that have been left untied by the current work.

\section{Analytical Calculations on Thin Graphs}

The (annealed) average of the partition function over all the thin graphs with $2 n$ vertices can be expressed as

$$
Z_{n}(\beta) \times N_{n}=\frac{1}{2 \pi i} \oint \frac{d \lambda}{\lambda^{2 n+1}} \int \frac{d \phi_{a} d \phi_{b}}{2 \pi \sqrt{\operatorname{det} K}} \exp (-S)
$$

where $N_{n}$ is the number of undecorated graphs with $2 n$ points,

$$
N_{n}=\left(\frac{1}{6}\right)^{2 n} \frac{(6 n-1) ! !}{(2 n) ! !}
$$

for $\phi^{3}$ graphs, and the action is identical to that of the matrix model in equ.(2).

If we rescale the fields $\phi \rightarrow \phi / \lambda$ and drop the overall factors that are not germane to the discussion then the large $n$ limit can be evaluated by calculating the saddle points of the action

$$
S=\frac{1}{2}\left(\phi_{a}^{2}+\phi_{b}^{2}\right)-\frac{1}{g} \phi_{a} \phi_{b}-\frac{1}{3}\left(\phi_{a}^{3}+\phi_{b}^{3}\right)
$$

A non-trivial phase structure can appear if there is a crossover in the dominant saddlepoint values, and this is what does in fact occur. In the $\phi^{3}$ model the solution to the saddle point equations are

$$
\phi_{a}, \phi_{b}=\frac{(g-1)}{g}
$$

\footnotetext{
${ }^{1}$ One can, however, think of the thin graphs as "infinite genus" surfaces with a maximal handle density [8].
} 


$$
\phi_{a}, \phi_{b}=\frac{1+g \pm \sqrt{(g+1)(g-3)}}{2 g}
$$

(along with a trivial zero solution, and a solution exchanging $\phi_{a}, \phi_{b}$ in the second case). The first set of solutions above is the high temperature unmagnetized phase and the second, which becomes dominant for $g>3$ is the low temperature magnetized phase, with the magnetization being given by [8]

$$
M=\frac{\phi_{a}^{3}-\phi_{b}^{3}}{\phi_{a}^{3}+\phi_{b}^{3}}
$$

If we denote the deviation from the critical coupling $\beta_{c}$ by $t=\left|\beta-\beta_{c}\right| / \beta_{c}$ then we see that we have meanfield like behaviour $M \simeq t^{1 / 2}$. The same is true for the other exponents which can easily be calculated using the results of $[8]$.

The saddle point equations can also be readily solved for the case of the Ising model on $\phi^{4}$ graphs, to give

$$
\begin{aligned}
\phi_{a}, \phi_{b} & =-\sqrt{1-1 / g} \\
\phi_{a} & =\frac{\left(\sqrt{1-\sqrt{1-4 / g^{2}}}\right)\left(1+\sqrt{1-4 / g^{2}}\right) g}{2 \sqrt{2}}, \\
\phi_{b} & =\frac{\sqrt{1-\sqrt{1-4 / g^{2}}}}{\sqrt{2}} .
\end{aligned}
$$

which indicate a phase transition at $g=2$, again with mean field exponents. If we pause for a second, and look back over the results we can see that we have reproduced the behaviour of the Ising model on Bethe lattices with 3 and 4 neighbours [10] with these results including, remarkably, the relevant critical temperatures. The correspondence between the Bethe lattice results and those on Feynman diagrams is unexpected because the Bethe lattice contains no loops, whereas the number of loops in the Feynman diagrams which can be calculated using standard methods is extensive [12], being $n+1$ for $\phi^{3}$ graphs with $2 n$ points for instance. The correspondance is not a fluke, it persists for the Ising model on $\phi^{5,6 . .}$ graphs and the corresponding Bethe lattices. Indeed, it remains true for the 3 and 4 state Potts models also, where we have

$$
g_{c}=\frac{z+p-2}{z-2}
$$

for the critical value of $g$ in a $p$-state Potts model on a Bethe lattice with $z$ neighbours [13]. The action for the 3 state Potts model on $\phi^{3}$ graphs is just

$$
S=\frac{1}{2}\left(\phi_{a}^{2}+\phi_{b}^{2}+\phi_{c}^{2}\right)-c\left(\phi_{a} \phi_{b}+\phi_{b} \phi_{c}+\phi_{c} \phi_{a}\right)-\frac{1}{3}\left(\phi_{a}^{3}+\phi_{b}^{3}+\phi_{c}^{3}\right)
$$

where $c$ is now given by

$$
c=\frac{1}{g+p-2}
$$

with $p=3$. This gives the following high and low temperature saddle point solutions

$$
\begin{aligned}
\phi_{a}, \phi_{b}, \phi_{c} & =1-2 c \\
\phi_{a} & =\frac{1+c+\sqrt{1-4 c-3 c^{2}-2 c \sqrt{1-4 c-4 c^{2}}}}{2} \\
\phi_{b} & =\frac{1+c-\sqrt{1-4 c-3 c^{2}-2 c \sqrt{1-4 c-4 c^{2}}}}{2} \\
\phi_{c} & =\frac{1+\sqrt{1-4 c-4 c^{2}}}{2}
\end{aligned}
$$


(along with permutations) which again display a transition at the Bethe lattice value of $g_{c}=4$ (ie $c=1 / 5)$. At the risk of belabouring the point the high and low temperature solutions for the 4 state Potts model on $\phi^{3}$ graphs

$$
\begin{aligned}
\phi_{a}, \phi_{b}, \phi_{c}, \phi_{d} & =1-3 c \\
\phi_{a}, \phi_{b}, \phi_{c}, \phi_{d} & =\frac{1+c \pm \sqrt{1-6 c-7 c^{2}}}{2},
\end{aligned}
$$

where two of the signs are to be taken positive and two negative in the second low temperature set, also fall into the pattern with $g_{c}=5(c=1 / 7)$.

It is thus clear that, at least for ferromagnetic bonds where there is no frustration, the Feynman diagrams offer an effective way of calculating with a closed version of the Bethe lattice. In fact, they might even be considered as more respectable than the Bethe lattice itself where one has to throw away the terms in the outermost shells to get a phase transition 10 . The correspondance between the diagrams and the Bethe lattice is underlined by the following observation, due to H. Hilhorst [11]: We can construct one of our Feynman diagrams in a Bethe-lattice-like manner by picking a seed point and following the standard procedure for constructing a Bethe lattice, but also allowing ourselves the possibility of joining branches to other branches at any step. This then gives the correct loop counting of $n+1$ in the $\phi^{3}$ model with $2 n$ vertices, once all the branches have been closed.

\section{Ferromagnet: Methodology and Simulations}

As a warmup exercise for simulations of the antiferromagnetic Ising model on Feynman diagrams we did some smaller scale simulations of the ferromagnetic model on $\phi^{3}$ and $\phi^{4}$ graphs. The generation of the graphs is a much easier exercise than generating the planar $\phi^{3}$ graphs used in $2 d$ gravity simulations as the absence of a constraint on the topology obviates the need to perform gymnastics with Tutte's algorithm. The simulation itself used standard finite size scaling techniques to determine the critical exponents. The absence of frustration in the ferromagnetic model allowed the use of the Wolff algorithm to give effective spin updating. We simulated on $\phi^{3}$ and $\phi^{4}$ graphs of sizes $250,500,1000,2500$ and 5000 points for various $\beta$ values, performing 20,000 measurements (after a suitable number of metropolis equilibration sweeps) with the number of Wolff sweeps per measurement being determined by the inverse of the mean cluster size. With ferromagnetic couplings and no frustration the quantities we use to characterize the transition, such as the energy and magnetization are all self-averaging, so we might expect to be able to obtain the correct critical behaviour using a single graph of each size, provided our graphs are not too small and we pick a representative graph. We shall see that this is, in fact, the case.

We commence our analysis of the results by looking at the crossing of the (unnormalized) Binder's cumulant for the magnetization

$$
U_{M}=\frac{<M^{4}>}{<M^{2}>^{2}}
$$

to locate the critical point. This provides a good test that we have managed to generate our graphs correctly. If we consider $\phi^{3}$ graphs first, the critical value of $g=3$ translates to $\beta_{c} \simeq 0.549$ and the crossing of the cumulant gives the estimate $\beta_{c} \simeq 0.547(2)$. Similarly on $\phi^{4}$ graphs $g=2$ translates to $\beta_{c} \simeq 0.347$ and the crossing of the cumulant gives the estimate $\beta_{c} \simeq 0.345(4)$. The quoted errors are the differences in the crossing points for different ratios of graph sizes. As a check we have done two complete sets of simulations with a different random graph of each size, and used all the possible combinations in the cumulant analysis. There was no appreciable variation between different graphs of the same size. The crossing of the cumulant is shown in Fig3.1 for three of the graph sizes used.

The value of the specific heat critical exponent $\alpha$ for the mean field Ising model is zero, signifying a discontinuity between a finite value at $\beta_{c}+$ and zero at $\beta_{c}$ - rather than the logarithmic divergence of the Onsager solution. If we look at Fig3.2 we see clear signs of this mean field behaviour in the form of the specific heat curve and a direct fit to the data is also consistent with $\alpha=0$. We can verify this by

\footnotetext{
${ }^{2}$ The lattice with the boundary terms included is often called the Cayley tree, and its thermodynamic behaviour is dominated by the terms on the boundary.
} 
looking at the scaling of the maximum slope of the magnetization cumulant which is expected to behave as

$$
\max \left(\frac{d U_{M}}{d \beta}\right) \simeq n^{\frac{1}{\nu d}}
$$

for a graph with $n$ vertices. This gives $\nu d=2.00(5)$ which, using the hyperscaling relation $\alpha=2-\nu d$, is again consistent with $\alpha=0$.

The value of $\nu d$ can also be fed into the finite size scaling relation for the vanishing of the magnetization at the critical point

$$
M \simeq n^{-\beta / \nu d}
$$

to extract a value for $\beta$. We find that $\beta / \nu d=0.24(1)$, so $\beta \simeq 1 / 2$ which is the mean field value. Similarly, the scaling of the linear susceptibility $\chi_{M}$ at the critical point

$$
\chi_{M}=\frac{d M}{d H} \simeq n^{\gamma / \nu d}
$$

can be fitted to extract the exponent $\gamma$, giving $\gamma=1.06(3)$ which is in respectable agreement with the mean field value of 1 .

We spare the reader the extraction of further exponents and the analysis of the $\phi^{4}$ results in a similar vein, as it is clear from the results already presented that the Ising model exhibits a mean field transition (as expected). The cumulant analysis also confirms that the transition occurs at the corresponding Bethe lattice transition points. Fortified by the good agreement between the analytical calculations and our simulations, we can now proceed to the case that really interests us, the antiferromagnet, to look for spin glass behaviour.

\section{Antiferromagnet: Calculations and Simulations}

In the absence of a $c=1$ barrier, we are at liberty to calculate with as many replicas as we can manage on thin graphs. It was pointed out in [8] that the saddle point equations for $k$ replicas of the Ising model on $\phi^{3}$ graphs admitted a factorized solution of the form

$$
\Phi=2^{1-k} \phi_{1} \phi_{2} \ldots \phi_{k}
$$

where the $\phi_{i}$ are individual solutions of the annealed, $k=1$, equation. The $k \rightarrow 0$ quenched free energy was equal to the annealed free energy, modulo finite size corrections. However, the entropy per spin calculated with this solution became negative for $g \simeq 0.211$ and higher moments of the partition function showed that the factorized solution no longer dominated

$$
\lim _{n \rightarrow \infty} \frac{1}{2 n} \log \overline{Z^{k}} \neq \lim _{n \rightarrow \infty} \frac{k}{2 n} \log \bar{Z}
$$

with the changeover point apparently converging to a finite value for $k$ increasing. Spin glass transitions are often signalled by the breakdown between quenched and annealed averages so, while this is not conclusive evidence for such a transition, it is certainly suggestive.

Despite the fact that only antiferromagnetic couplings are present in the model, there is certainly frustration due to the loops. Indeed, a variational calculation by de Seze some years ago [14] noted that an Ising antiferromagnet with dilute bond disorder would have a spin glass phase if the undiluted version of the model did not allow antiferromagnetic order at $T=0$. The hamiltonian in this case was

$$
H=-\sum_{i j} J_{i j} \sigma_{i} \sigma_{j}
$$

where the sum was over all $i, j$ and the $J_{i j}$ were distributed (in a quenched ensemble) according to

$$
P\left(J_{i j}\right)=c \delta\left(J_{i j}+J\right)+(1-c) \delta\left(J_{i j}\right)
$$


which has the effect of killing a fraction $1-c$ of the bonds in any given realization. The model we consider on Feynman diagrams could be cast in rather similar form, as we have some probability that two vertices are connected for a given graph, so viewed in this light spin-glass like behaviour is not so surprising.

Although we would no longer expect such close similarity with the standard Bethe lattice as frustration has highlighted the role of the loops, there have been previous simulations of "closed" versions of the Bethe lattice [15] that might be expected to be a closer approximation. These considered mixed ferromagnetic and antiferromagnetic bonds on a Bethe lattice where the branches were closed in two ways - either with fixed boundary conditions, where no spin glass behaviour was seen, or with pairing between different branches in a given shell, which did display signs of a spin glass phase. Unfortunately purely antiferromagnetic couplings were not considered, which would have allowed a more direct comparison with our results as the Feynman diagrams are effectively a multi-shell generalization of the paired closure.

Our tactics for simulating the antiferromagnet were rather similar to those in [15]. We took an explicitly quenched average by generating $O(100)$ different Feynman diagrams for each of the sizes that we simulated, 100, 250,500 and 1000 points. The number of lattices simulated is justified a posteriori by checking that the results for non-self averaging observables, such as the overlap described in the next paragraph, look reasonable. We used a massively parallel processor (the Intel Paragon) to run the different lattices concurrently and perform the requisite quenched averaging over the observables during the course of the simulation. For the sake of simplicity we made no attempt to use either multicanonical, simulated tempering, or replica cluster simulation schemes which are applicable, usually with some effort, to frustrated systems such as ours [16] but stuck to the metropolis algorithm. At each $\beta$ point we employed 500,000 simulated annealing sweeps to cool the system followed by 200,000 production sweeps with a measurement every 10th sweep. Each sweep consisted of a complete metropolis update of the lattice.

In addition to all of the standard thermodynamic variables that we measured in the ferromagnetic case we paid particular attention to the overlap between two replicas of the Ising model on a given graph, as non trivial behaviour in the overlap distribution is often the sign of a spin glass phase. The overlap is defined in the standard fashion by putting two independent replicas on each graph in the ensemble

$$
q=\frac{1}{n} \sum_{i=1}^{n} \sigma_{i} \tau_{i}
$$

where $\sigma_{i}$ is the spin at site $i$ in one replica, and $\tau_{i}$ is the spin at site $i$ in the other. We then measure

$$
P_{n}(q)=\left[\left\langle\delta\left(q-1 / n \sum \sigma_{i} \tau_{i}\right)\right\rangle\right]
$$

where [ ] denotes the quenched graph average and $<>$ the thermal average, to get a histogram of the overlap probability.

$P(q)$ is known to be strongly non-self-averaging in soluble models such as the Sherrington Kirkpatrick (SK) model [17] so the quenched average is vital, given the strong variation between individual graphs. A useful heursitic check in the simulations in to make sure that $P(q)$ is symmetric about $q=0$ as $P(q)=P(-q)$ analytically. If this is not the case the data is almost certainly not equilibrated. We have also measured the autocorrelation times in the measurements of $P(q)$ in order to help judge their reliability.

It is known in the SK model that $P(q)$ is related to the Parisi order parameter by $P(q)=d x(q) / d q$ [18], but in principle there are an infinite number of order parameters generalizing the overlap in short range models 19 . It has also been observed that $P(q)$ can produce misleading results in short range spin glasses, which might not exhibit the coexistence of infinitely many pure states that is seen in the mean field solution of the SK model [20], but rather have a two phase "droplet" structure. Recent numerical work [21] on the nearest neighbour four dimensional $\pm J$ Ising spin glass, however, seems to support a mean field like picture so the question is far from settled. In any case, given that the genuine spin glass with nearest neighbour $\pm J$ couplings on the Bethe lattice behaves in an SK like way with the appropriate boundary conditions [23], it is not unreasonable to expect that $P(q)$ may be an appropriate tool to use 
for the Feynman diagrams we consider here. In addition, observables such as the spin glass susceptibility

$$
\chi_{s g}=\frac{1}{n} \sum_{i j}\left[\left\langle\sigma_{i} \sigma_{j}\right\rangle^{2}\right]
$$

may be calculated from the moments of $P(q)$

$$
\chi_{s g}=n \int q^{2} P_{n}(q) d q .
$$

We also measured directly the Binder's cumulant associated with $q$

$$
U_{s g}=\frac{\left\langle q^{4}\right\rangle}{\left\langle q^{2}\right\rangle^{2}}
$$

whose crossing points for differently sized lattices might be expected to pinpoint any spin glass transition point, in a similar manner to the cumulant for the magnetization in ferromagnetic transitions.

As in the ferromagnetic case, we commence our analysis by looking for the crossing point of the appropriate cumulant, $U_{s g}$. The results for this are much less accurate than for the ferromagnetic transition, both because of the smaller lattice sizes simulated and the non-self averaging nature of quantities that concern the overlap. Nonetheless, we obtain a crossing for three of the four lattice sizes, indicating that there is a spin glass transition with $\beta_{c} \simeq-0.94(2)$, where the error is again from the different intercepts for different ratios of lattice sizes. This translates to $g \simeq 0.15$, which is rather smaller than the value indicated by the negative entropy in $[8]\left(g \simeq 0.211\right.$ or $\left.\beta_{c} \simeq-0.78\right)$. However, using the high temperature solution to extract the entropy in the SK model 20 gives an expression that becomes negative inside the spin glass phase rather than at the transition point as in the random energy model [22]. In [8] the entropy changes sign in what seems to be the paramagnetic phase from our simulations, but the apparent inversion here with respect to the SK model may be due to the purely antiferromagnetic couplings. We show the crossing of the cumulant in Fig4.1, where we have dropped the results from the $N=500$ lattices that (narrowly) failed to cross the others in the plot.

It is known that the specific heat displays a cusp at $\beta_{c}$ in the SK model corresponding to an exponent $\alpha=-1$, but if we look at our data in Fig4.2 we see that, although there is a cusp it appears to be in the region of $\beta \simeq-0.84$. However, the specific heat estimations of the critical coupling need to be extrapolated to infinite volume to compare with the cumulant results. To do this we need to extract a value for the combination $\nu d$ from our data 3 which can be done by regarding the scaling of the maximum slope of $U_{s g}$ which, just as for $U_{M}$, is expected to behave as

$$
\max \left(\frac{d U_{s g}}{d \beta}\right) \simeq n^{\frac{1}{\nu d}} .
$$

We find $\nu d=2.8(2)$, which is consistent with the cusp displayed by the specific heat as the hyperscaling relation $\alpha=2-\nu d$ still holds. It is also compatible with the mean field SK value $\alpha=-1$. If we now use our value of $\nu d$ to extrapolate the $\beta_{c}$ 's for the specific heat to infinite volume we find $\beta_{c}(\infty) \simeq-0.88(2)$, which is less negative than the value from the cumulant crossing. The quoted error for the critical coupling determined from the specific heat cusp is probably too optimistic, given it is the result of a fit first to $\nu d$ followed by a linear interpolation of the estimated cusp positions, so the cumulant crossing value is certainly more reliable.

The linear susceptibility $\chi_{M}=d M / d H$ does not diverge at a spin glass transition point, but is expected to display a cusp similar to that in the specific heat. In Fig4.3 we plot $\chi_{M}$ and see clear evidence of a cusp although it is noteworthy that this is considerably displaced from $\beta \simeq-0.95$. The finite size scaling does not give such a convincing shift back towards this value as in the specific heat case, but the quality of the data is poorer. From the spin glass point of view the more interesting susceptibility is $\chi_{s g}$ defined in equ.(29), which we measured both directly and using using equ.(30). The data from the

\footnotetext{
${ }^{3}$ Although $d$ may be rather ill-defined on our diagrams, the combination is not.
} 
direct measurements is plotted in Fig4.4. $\chi_{s g}$ is expected to diverge at a spin glass transition, in contrast to $\chi_{M}$, so there will be a finite size scaling relation of the form

$$
\chi_{s g} \simeq n^{\gamma / \nu d}
$$

analogously to equ.(22). Fitting the data from both ways of extracting $\chi_{s g}$ we find $\gamma=1.3(2)$, where the error bar does not take account of the uncertainty in determining the critical point. This value is still marginally compatible with the SK value of 1 .

Looking directly at the histograms of $P(q)$ also gives indications of a transition around $\beta \simeq-0.95$. In Figs4.5-4.6, we show the histograms for $\beta=-0.1$, deep in the paramagnetic phase and at $\beta=-1.2$, below the transition point. We have plotted the histograms without the error bars coming from the quenched average (which are not particularly large) for clarity. The histograms in Fig 4.5 are clearly gaussian in shape, as expected for a paramagnetic phase and the width of the gaussians for differently size graphs is in reasonable agreement with "delta-function" like behaviour, namely $\sigma^{2} \simeq n^{-1}$. As the region around $\beta \simeq-0.95$ is approached the histograms broaden considerably and begin to show signs of a dip at the origin. Once we are in the putative spin glass phase the histograms look generically like that in Fig4.6 for $\beta=-1.2$. Comparing with histograms for the different lattice sizes shows that the peak at $q \simeq 0.5$ is sharpening with increasing lattice size and that the "tail" stretching down to $q=0$ remains stable. These results suggest that the behaviour is strikingly similar to the SK spinglass, with a delta function at finite $q$ adjoined to a continuous tail down to $q=0$ in the infinite volume limit. This also means that the distribution of $P(q)$ takes on a form very similar to that seen on a Bethe lattice spin-glass with closed boundary conditions in the simulations in 15. Once we are some distance below $\beta \simeq-0.95$ the autocorrelation times for the overlap become very large and the histograms begin to lose their symmetry and roughen, suggesting that the data has failed to equilibrate, a behaviour often seen in spin glass simulations.

From these results the Ising antiferromagnet on Feynman diagrams would appear to possess a spin glass phase at sufficiently negative $\beta$ with similar properties to the SK model. It is thus behaving in a mean field like manner and all the paraphernelia of replica symmetry breaking might be expected to be applicable.

\section{Antiferromagnet on Fat Graphs}

As we indicated in the introduction there is nothing to stop us simulating the Ising model on a quenched ensemble of fat graphs, even though such a system is currently not accesssible to reliable analytical calculations. The simulations of an Ising ferromagnet on such an ensemble in [7] encountered no obvious pitfalls and gave results that were very similar to the annealed ensemble of dynamical graphs. One might expect quenched and annealed averages to give rather similar results when frustration is not playing a major role, so the antiferromagnet may well be a different story entirely. A simulation of an Ising antiferromagnet on quenched fat graphs is of interest because the graphs involved are no longer of an "infinite-dimensional" mean field type. Indeed, it appears that the fractal dimension of the graphs is 3 or 4 (depending on the method of definition) [24], and 3 may be the lower critical dimension of the spin-glass transition. It might be possible to look analytically at the change, if any, from the mean field behaviour on $N=1$ thin graphs as the size of the matrices $N$ is increased to fatten the graphs. Such an approach has already provided surprisingly good values for critical exponents and couplings in the one matrix model by extrapolation from quite small $N$ [25].

We are less ambitious here and simply conduct a trial simulation of the Ising model on $O(100)$ planar $\phi^{3}$ graphs with 250 vertices in each. The loop distribution is calculable using the methods of [2] and is strongly peaked at small $(3,4 .$.$) loops. This means that by comparison with the thin graphs, where the$ loops are in general rather large and the local structure tree-like, frustration is likely to play a stronger role. We use the same number of graphs and simulated annealing sweeps in the simulation as in the thin graph simulations of the preceding section. We find that the histograms of $P(q)$ begin to deviate strongly from a gaussian shape in the region of $\beta=-1.5$ and become very irregular and assymetric for $\beta<-2.0$. This is a signal either that we have not equilibrated the system or failed to include enough graphs in the 
quenched average for these $\beta$ values. Increasing the simulated annealing steps by a factor of four makes little difference to the roughness of the $P(q)$. The associated autocorrelation times for the measurements of $q$ also rapidly become astronomical below $\beta=-1.5$.

In the region of $\beta=-1.5$ the observed histograms are rather similar to those in the region of $\beta=-0.95$ on thin graphs, being perhaps more irregular. This would mean that one has to work harder on fat graphs than on thin graphs to obtain reliable numerical results. One of the histograms for $P(q)$ at $\beta=-1.9$ for the fat graph is shown in Fig5.1, where we have included a histogram of $P(q)$ at $\beta=-1.3$ on a thin graph of 250 vertices for comparison. Allowing for the differing "distances" from the apparent critical values and remembering that a size of 250 on a fat graph may not be equivalent to a size of 250 on a thin graph, the two distributions are very similar in form.

As we have only simulated a single graph size, we make no attempt to conduct a serious scaling analysis with our current fat graph data and content ourselves with some qualitative observations: The non-trivial distribution of $P(q)$ that has been observed, and the large autocorrelation times suggest that something non-trivial is still going on for the antiferromagnet on fat graphs for sufficiently negative $\beta$. If the form of $P(q)$ persisted on larger graphs it would be suggestive of mean field like behaviour, given its similarity to the thin graph distribution and hence that of the SK model.

\section{$6 \quad$ Higher State Potts Models}

We have thus seen that the Ising antiferromagnet on $\phi^{3}$ graphs displays a non-gaussian $P(q)$ for sufficiently negative couplings, which strongly resembles those seen in 115 for the Ising spin glass on a Bethe lattice with closed boundary conditions, and those for the SK model. It would appear that the model is closer to these mean-field systems than short range spin glasses. Given this it is of interest to enquire whether higher state Potts models also display non-trivial $P(q)$ on Feynman diagrams for purely negative couplings. It is not immediately obvious that they will, as it is easier to "unfrustrate" a given loop if we have more colours. In addition, genuine Potts glasses ( $\pm J$ or Gaussian distributed couplings) have a completely different order parameter distribution to the Ising spin glass in both infinite range models and on Bethe lattices [26, 27. In the infinite range (mean field) case they display two transition temperatures, with a $P(q)$ that is still a delta function below the first and two delta functions joined by a continuous portion below the second. On the Bethe lattice the form of $P(q)$ depends on the bond distribution and the number of neighbours, for $\pm J$ bonds in the 3 state Potts model for instance there is a delta-function when the number of neighbours $z \geq 4$ and a continuous distribution for $z=3$. For the 4 state Potts model there is only a delta function [27]. For more than four states in both cases the phase transition displays some first order properties.

For p-state Potts models the overlap can be defined as

$$
q=\frac{1}{n} \sum_{i=1}^{n}\left(p \delta_{\sigma_{i}, \tau_{i}}-1\right)
$$

which is arranged to be zero in the uncorrelated case $\delta_{\sigma_{i}, \tau_{i}} \simeq 1 / p$. This can be measured just as in the Ising simulations. A quick test simulation of the 3 state model on $\phi^{3}$ graphs reveals that the distribution of $P(q)$ does not appear to change from the paramagnetic form even for large negative couplings, suggesting there is no spinglass phase at all. The intuition that there is now enough liberty to relieve the frustration can, be substantiated by Brook's theorem from graph theory [28]: If $\mathrm{G}$ is a connected graph with maximal vertex valency $z>2$, and if $\mathrm{G}$ is not complete, then the chromatic number $\chi$ the minimum number of colours it takes so that no adjacent vertices are the same colour, of $\mathrm{G}$ is $z$. In this language we have a 3-regular graph that is not complete, so $\chi=3$ and we can avoid frustration completely with 3 colours. By the same token, we would not expect any spinglass like behaviour on $\phi^{z}$ graphs for $p \geq z$ state Potts models with purely negative couplings as $\chi=z$ for these.

Chromatic numbers for more general random graphs are rather large, though some asymptotic bounds are known, that are in accordance with simulations of antiferromagnetic Potts models [29]. There are

\footnotetext{
${ }^{4}$ Not to be confused with either of the susceptibilities $\chi_{M}, \chi_{s g}$ earlier!

${ }^{5}$ We would like to thank N. Gilbert for pointing this out to us.
} 
of course, particular cases such as the solution of the celebrated four colour problem, which states that $\chi=4$ for any planar map [30]. $\chi=2$ trivially for a planar square lattice and a three colouring algorithm also exists as a consequence of the solution of the six vertex model [31. Surprisingly, another quick test simulation for a 3 state Potts model with purely negative couplings on $\phi^{4}$ Feynman diagrams (so neither Brooks' theorem nor the four-colour theorem apply) also produced distributions that did not change from the paramagnetic form with decreasing beta. It would be interesting to confirm this behaviour and explore it further for different $p$ and graphs.

A brief summary of whether or not a spin glass transition appears to occur in the cases discussed above $(\sqrt{ })$ or not $(\times)$ is given in the table below. We have left the $p=3$ model on thin $\phi^{4}$ graphs undecided.

\begin{tabular}{|c|c|c|c|}
\hline$p=2$ & thin & $\phi^{3}$ & $\sqrt{ }$ \\
\hline$p=2$ & fat & $\phi^{3}$ & $\sqrt{ }$ \\
\hline$p=3$ & fat,thin & $\phi^{3}$ & $\times$ \\
\hline$p=3$ & thin & $\phi^{4}$ & $?$ \\
\hline$p=4$ & fat & any & $\times$ \\
\hline$p \geq z$ & fat,thin & $\phi^{z}$ & $\times$ \\
\hline
\end{tabular}

Table 1: Summary of whether a spin glass transition appears $\sqrt{ }$ or not $\times$.

\section{Conclusions}

We have seen analytically in section 2 that both Ising and higher state Potts models on Feynman diagrams reproduce the Bethe lattice mean field behaviour, even down to the same critical couplings for Bethe lattices with the same number of nearest neighbours. In section 3 we saw that the self averaging nature of the quantities that characterize the transition, such as the energy and the magnetization, meant that we could obtain numerically respectable results with a single graph. These were in good accord with the analytical calculations. The net conclusion is thus that, at least as far as ferromagnetic couplings are concerned, the Ising and Potts models do not "see" the loops that make the difference between the Bethe lattice and the Feynman diagrams.

These loops $d o$, however, play a role once antiferromagnetic couplings are introduced. The calculations of [8], suggesting a spinglass phase in the Ising model for sufficiently negative coupling, are supported by the non-trivial $P(q)$ 's that were observed in section 4 . The quenched average over different Feynman diagrams was obligatory in this case because of the non-self-averaging properties of $P(q)$. The results seen on the Feynman diagrams are strongly reminiscent of the Bethe lattice with closed boundary conditions that was simulated in [15]. The form of the $P(q)$ for the Ising antiferromagnet suggests that we are closer to a mean field picture of spin glass behaviour than a droplet one on Feynman diagrams, again much like the Bethe lattice, and in accordance with earlier calculations for finite connectivity random lattices. The critical exponents we extracted were also all in reasonable agreement with those in the mean field SK model.

In section 5 we looked briefly at the Ising antiferromagnet on planar or fat graphs, finding that the distribution of $P(q)$ was rather similar to that on a thin graph of a similar size. The antiferromagnetic higher state Potts models that we also briefly looked at in section 6 show interesting behaviour, and there are some intriguing links with colouring problems in graph theory.

The utility of Feynman diagrams as a tool in analytical calculations with random graphs of finite connectivity is thus clear. The close connection between the Feynman diagrams and the Bethe lattice is somewhat unexpected at first sight, but persists even for the antiferromagnet and closed versions of the Bethe lattice. Both the ferromagnet and antiferromagnet have displayed essentially mean field behaviour on thin graphs. The simulations of [7] suggest that this is not the case for the ferromagnet on fat graphs, so it would be worth pursuing the simulations of the antiferromagnet on fat graphs to look for non mean field behaviour and exponents. We have not explored issues such as the scaling of $P(q)$, the ultrametricity 
(or otherwise) of the distribution of states and the response to external fields for the antiferromagnet in the current work, but we hope to return to these in future.

There are also various direct generalizations that we have not had the time to explore in the current paper. A first is to look at "real" spinglasses with either $\pm J$ or gaussian couplings on the Feynman diagrams to compare with previous Bethe lattice results for such models. A second obvious extension is to look at other models such as the $X Y$ model on thin graphs, for which some analytical results already exist in the unfrustrated case [32]. It might also be worthwhile to implement some of the more advanced simulation algorithms on the models described here. There are clearly plenty of interesting questions to be addressed regarding quenched disorder on both thin and fat graphs.

\section{Acknowledgements}

The ferromagnetic simulations reported in this paper were carried out on workstations at Heriot-Watt University, LPTHE Orsay and the University of Colorado. The antiferromagnetic simulations were carried out on the Front Range Consortium's 208-node Intel Paragon located at NOAA/FSL in Boulder. Some of the Binder's cumulant analysis was carried out using programs written by A. Krzywicki. DAJ was supported at Orsay by an EEC Human Capital and Mobility Fellowship and an Alliance grant and would like to thank A. Krzywicki and H. Hilhorst for various useful conversations there. CFB is supported by DOE under contract DE-FG02-91ER40672, by NSF Grand Challenge Applications Group Grant ASC9217394 and by NASA HPCC Group Grant NAG5-2218.

\section{References}

[1] V. A. Kazakov, Phys. Lett. A119 (1986) 140;

D.V. Boulatov and V.A. Kazakov, Phys. Lett. B186 (1987) 379;

Z. Burda and J. Jurkiewicz, Acta Physica Polonica B20 (1989) 949.

[2] E. Brezin, C. Itzykson, G. Parisi and J.B. Zuber, Commun. Math. Phys. 59 (1978) 35;

M.L. Mehta, Commun. Math. Phys. 79 (1981) 327.

[3] Z. Burda and J. Jurkiewicz, Phys. Lett. B214 (1988) 425.

[4] V.G. Knizhnik, A.M. Polyakov and A.B. Zamolodchikov, Mod. Phys. Lett. A3 (1988) 819;

F. David, Mod. Phys. Lett. A3 (1988) 1651;

J. Distler and H. Kawai, Nucl. Phys. B321 (1989) 509.

[5] J. Jurkiewicz, A. Krzywicki, B. Petersson and B. Soderberg, Phys. Lett. B213 (1988) 511;

R. Ben-Av, J. Kinar and S. Solomon, Nucl. Phys. B ( Proc. Suppl.) 20 (1991) 711;

S.M. Catterall, J.B. Kogut and R.L. Renken, Phys. Rev. D45 (1992) 2957;

C. Baillie and D. Johnston, Mod. Phys. Lett. A7 (1992) 1519;

J. Ambjørn, B. Durhuus, T. Jonsson and G. Thorleifsson, Nucl. Phys. B398 (1992) 568.

[6] S.F. Edwards and P.W. Anderson, J. Phys. F5 (1975) 965;

M. Mezard, G. Parisi and M. Virasoro, "Spin Glass Theory and Beyond" World Scientific, 1987;

K. Binder and A.P. Young, Rev. Mod. Phys. 58 (1986) 801.

[7] D. Johnston, Phys. Lett. B277 (1992) 405;

C. Baillie, K. Hawick and D. Johnston, Phys. Lett. B328 (1994) 251.

[8] C. Bachas, C. de Calan and P. Petropoulos, "Quenched Random Graphs", Ecole Polytechnique Preprint CPTH-A264.1093, hep-th 9405068. 
[9] J. Banavar, D. Sherrington and N Sourlas, J. Phys. A 20 (1987) L1;

P. Mottishaw, Europhys. Lett. 4 (1987) 333;

C. de Dominicis and Y. Goldschmidt, J. Phys. A22 (1989) L775. P-Y Lai and Y. Goldschmidt, J. Phys. A23 (1990) L775.

[10] H. A. Bethe, Proc. Roy. Soc. A 150 (1935) 552;

C. Domb, Advan. Phys. 9 (1960) 145;

T. P. Eggarter, Phys. Rev. B9 (1974) 2989;

E. Muller-Hartmann and J. Zittartz, Phys. Rev. Lett. 33 (1974) 893.

[11] H. Hilhorst, private communication.

[12] J. Binney, N. Dowrick, A. Fisher and M. Newman, "The Theory of Critical Phenomena", O.U.P, Oxford 1992.

[13] R. Baxter, "Exactly Solved Models in Statistical Mechanics", Academic Press, London 1982.

[14] L. de Seze, J. Phys. C10 (1977) L353.

[15] P-Y Lai and Y. Goldschmidt, J. Phys. A22 (1989) 399.

[16] R. Swendsen and J-S. Wang, Phys. Rev. Lett. 57 (1986) 2607;

B. Berg and T. Celik, Phys. rev. Lett. 69 (1992) 2992;

E. Marinari and G. Parisi, Europhys. Lett. 19 (1992) 451.

[17] D. Sherrington and S. Kirkpatrick, Phys. Rev. Lett. 35 (1975) 35.

[18] G. Parisi, Phys. Rev. Lett. 50 (1983) 1946.

[19] L. Viana and A. Bray, J. Phys. C 18 (1985) 3037.

[20] D. Fisher and D. Huse, J. Phys. A 20 (1987) L997,L1005.

[21] J. Ciria, G. Parisi and F. Ritort, J. Phys. A 26 (1993) 6711,6731.

[22] B. Derrida, Phys. Rev. B24 (1981) 2613.

[23] D. Thouless, Phys. Rev. Lett. 56 (1986) 1082;

J. Chayes, L. Chayes, P Sethna and D. Thouless, Comm. Math Phys. 106 (1986) 41.

[24] H. Kawai, N. Kawamoto and Y. Watakibi, Nucl. B306 (1990) 19.

[25] S. Hikami, Physica A204 (1994), hep-th 9309005.

[26] D. Gross, I. Kanter and H. Sompolinsky, Phys. Rev. Lett. 55 (1985) 304.

[27] Y. Goldschmidt, Europhys. Lett. 6 (1988) 7.

[28] R. Wilson, "Introduction to Graph Theory", 3rd edition Longman 1985

[29] P-Y. Lai and Y. Goldschmidt, J. Stat. Phys. 48 (1987) 513;

B. Bollobas and P. Erdos, Math. Proc. Camb. Phil. Soc. 80 (1976) 419.

[30] K. Appel and W. Haken, Bull. Am. Math. Soc. 82 (1976) 711.

[31] E. Lieb, Phys. Rev. 162 (1963) 162.

[32] N. Dorey and P. Kurzepa, Phys. Lett. B295 (1992) 51. 


\section{Figure Captions}

Fig. 3.1 The crossover in Binder's cumulant for the magnetization in the ferromagnetic model for three representative $\phi^{3}$ graphs.

Fig. 3.2 The specific heat in the ferromagnetic model, displaying mean field like behaviour.

Fig. 4.1 The crossover in Binder's cumulant for the overlap in the antiferromagnetic model on $\phi^{3}$ graphs with $N=100,250,1000$.

Fig. 4.2 The specific heat in the antiferromagnetic model, which shows a characteristic cusp.

Fig. 4.3 The linear susceptibility $\chi_{M}=d M / d H$ for the antiferromagnet.

Fig. 4.4 The spin glass susceptibility $\chi_{s g}$ for the antiferromagnet.

Fig. 4.5 A histogram of $P(q)$ at $\beta=-0.1$ which is deep in the paramagnetic phase. We have only plotted the range $q=0,0.25$ and histograms for $n=250,1000$ for clarity.

Fig. 4.6 A histogram of $P(q)$ at $\beta=-1.2$, in the conjectured spin glass phase. We have plotted three graph sizes to show the sharpening of the peak and the stability of the tail.

Fig. 5.1 A histogram of $P(q)$ at $\beta=-1.9$ for the antiferromagnet on a $\phi^{3}$ planar (fat) graph with 250 vertices. A histogram of $P(q)$ at $\beta=-1.3$ for a thin graph of similar size is also shown for comparison. 


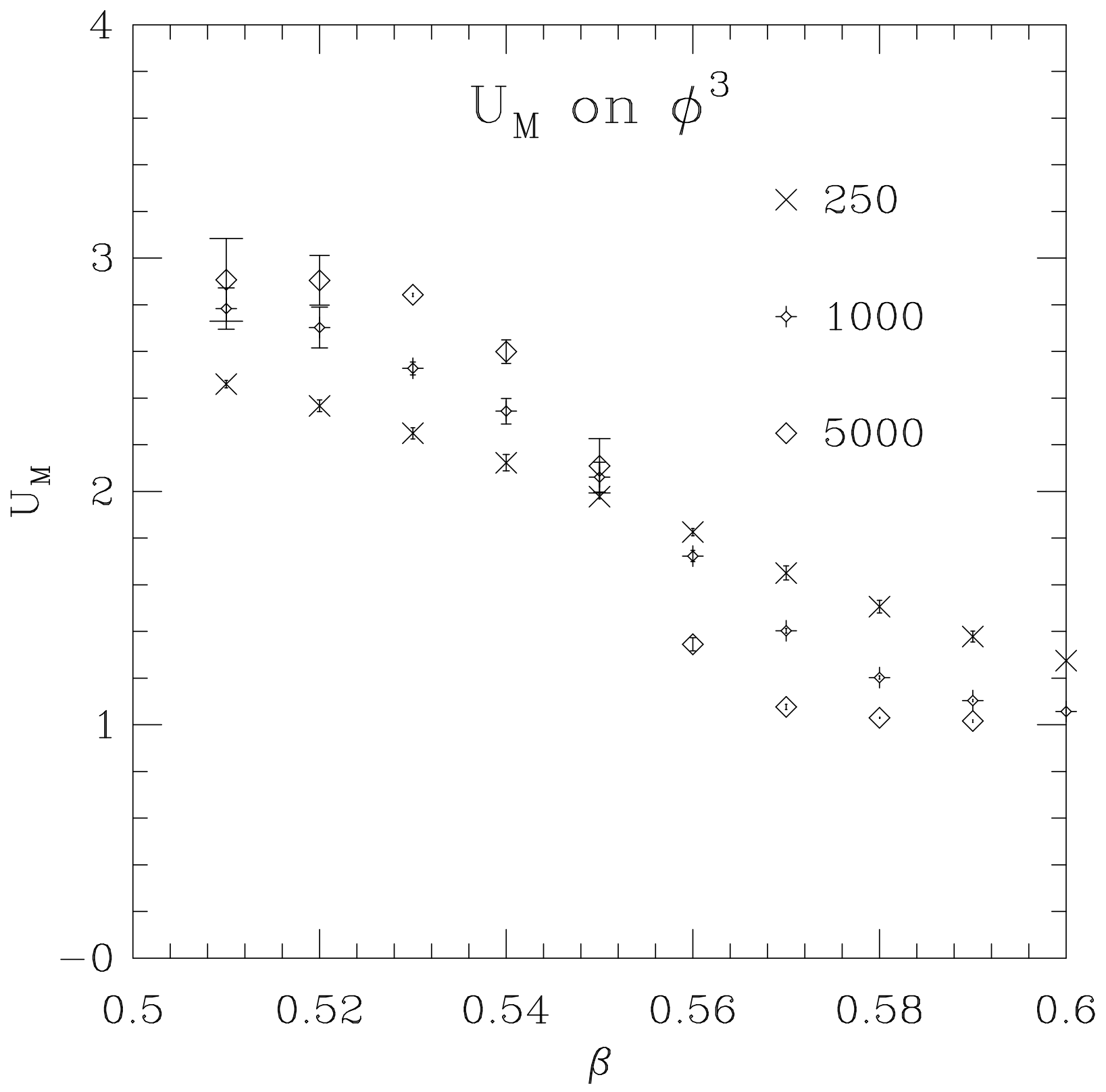




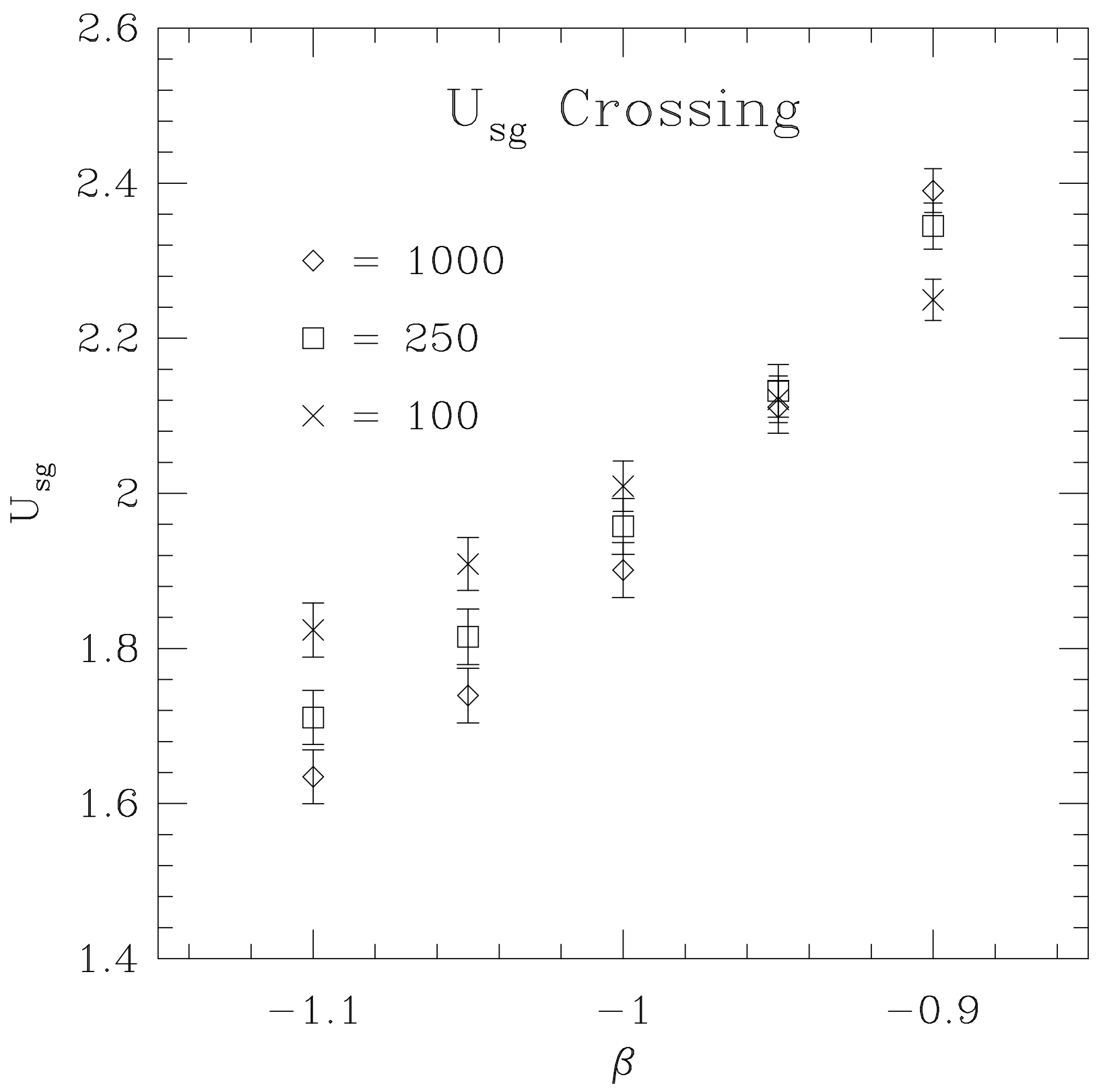




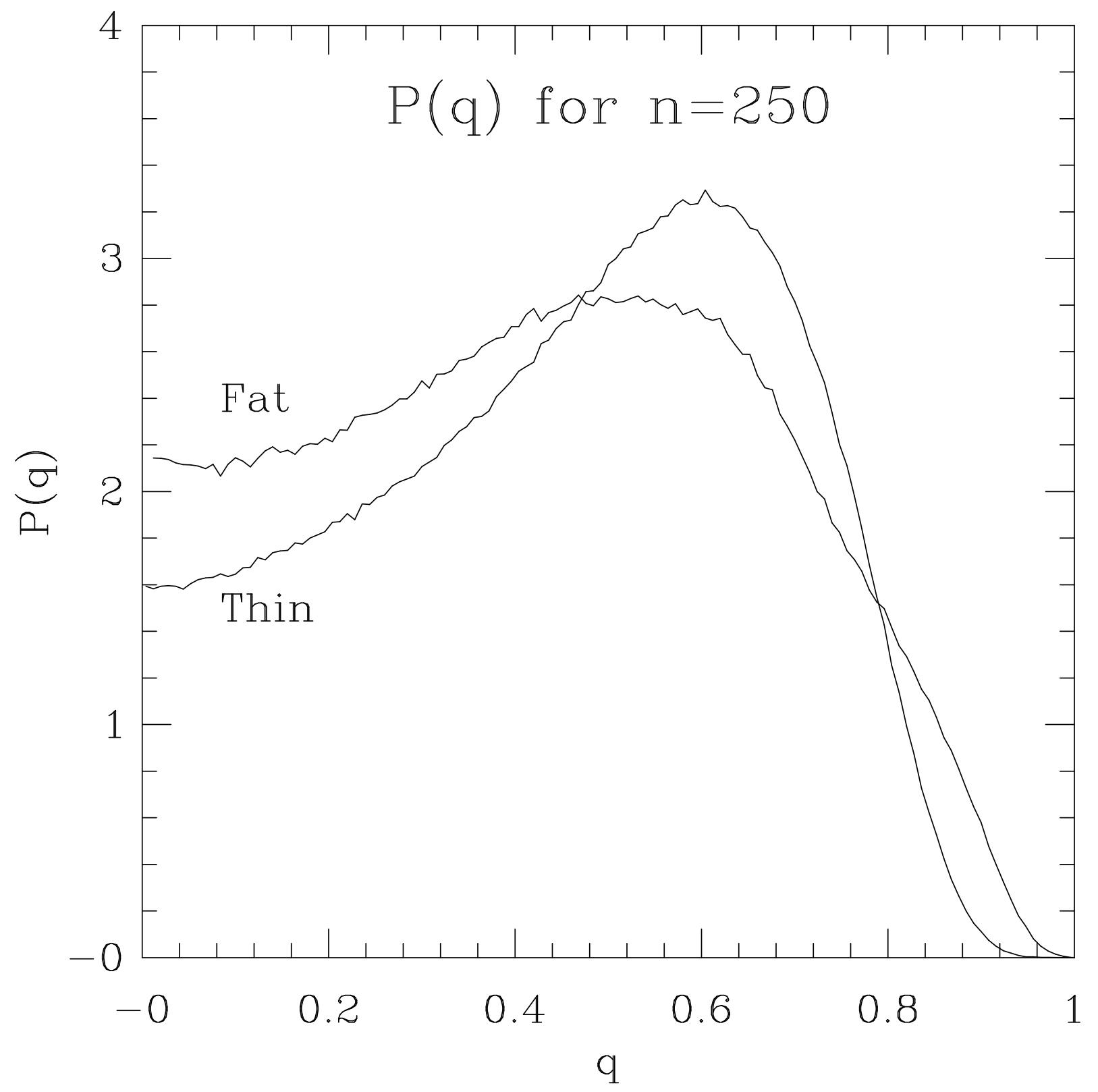


This figure "fig1-1.png" is available in "png" format from: http://arxiv.org/ps/hep-lat/9407024v1 
This figure "fig2-1.png" is available in "png" format from: http://arxiv.org/ps/hep-lat/9407024v1 


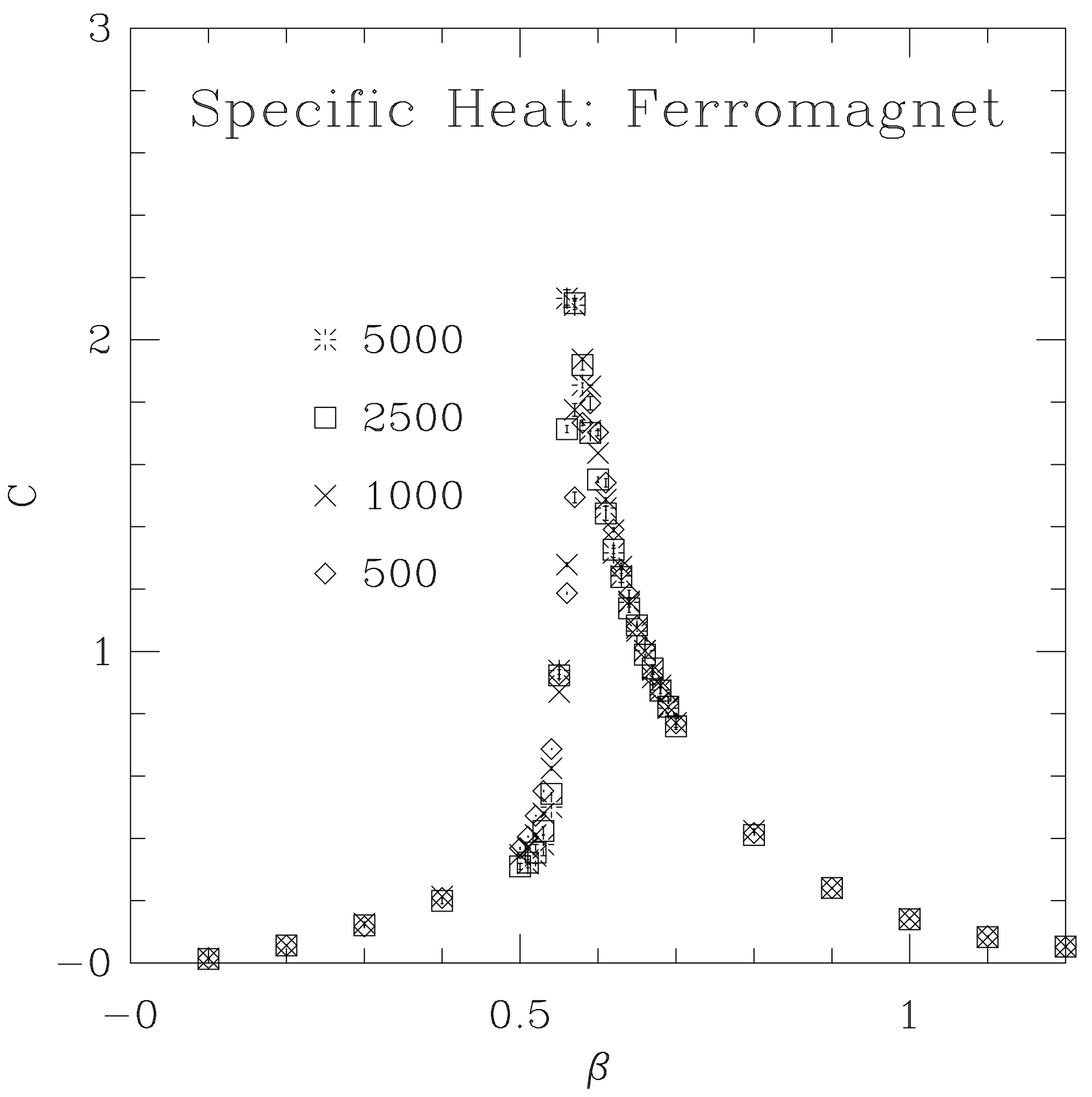




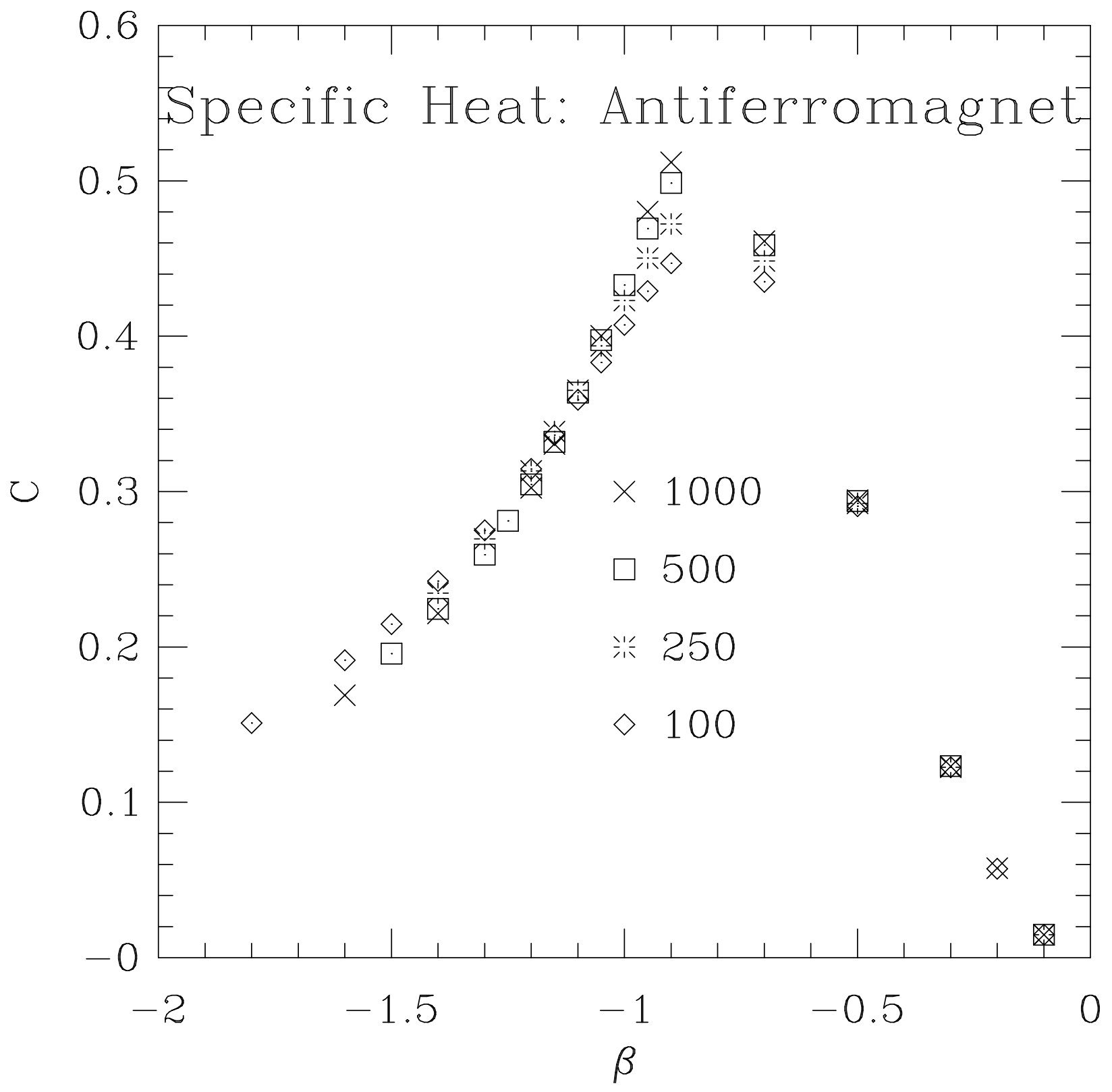


This figure "fig1-2.png" is available in "png" format from: http://arxiv.org/ps/hep-lat/9407024v1 
This figure "fig2-2.png" is available in "png" format from: http://arxiv.org/ps/hep-lat/9407024v1 


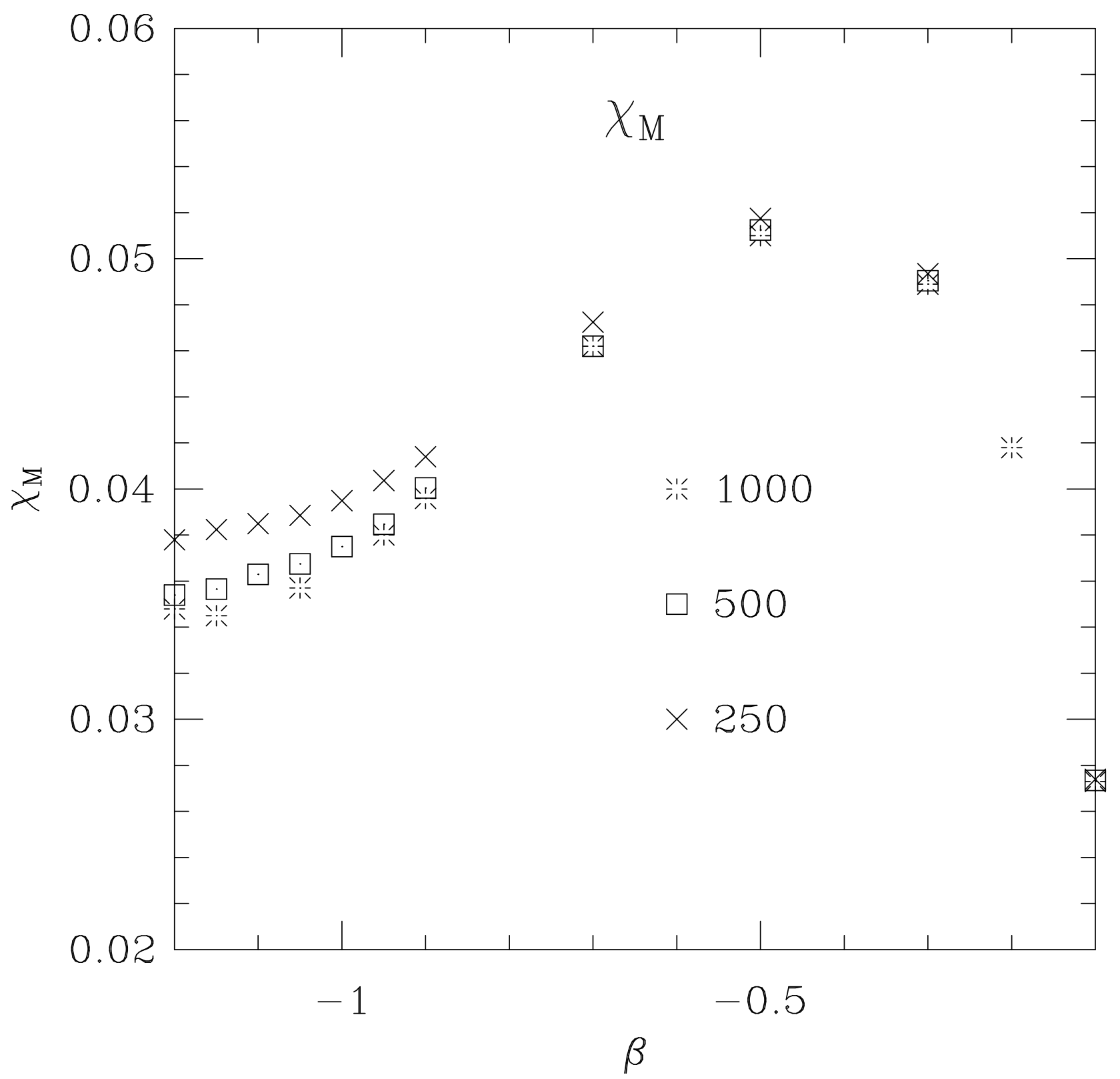


This figure "fig1-3.png" is available in "png" format from: http://arxiv.org/ps/hep-lat/9407024v1 
This figure "fig2-3.png" is available in "png" format from: http://arxiv.org/ps/hep-lat/9407024v1 


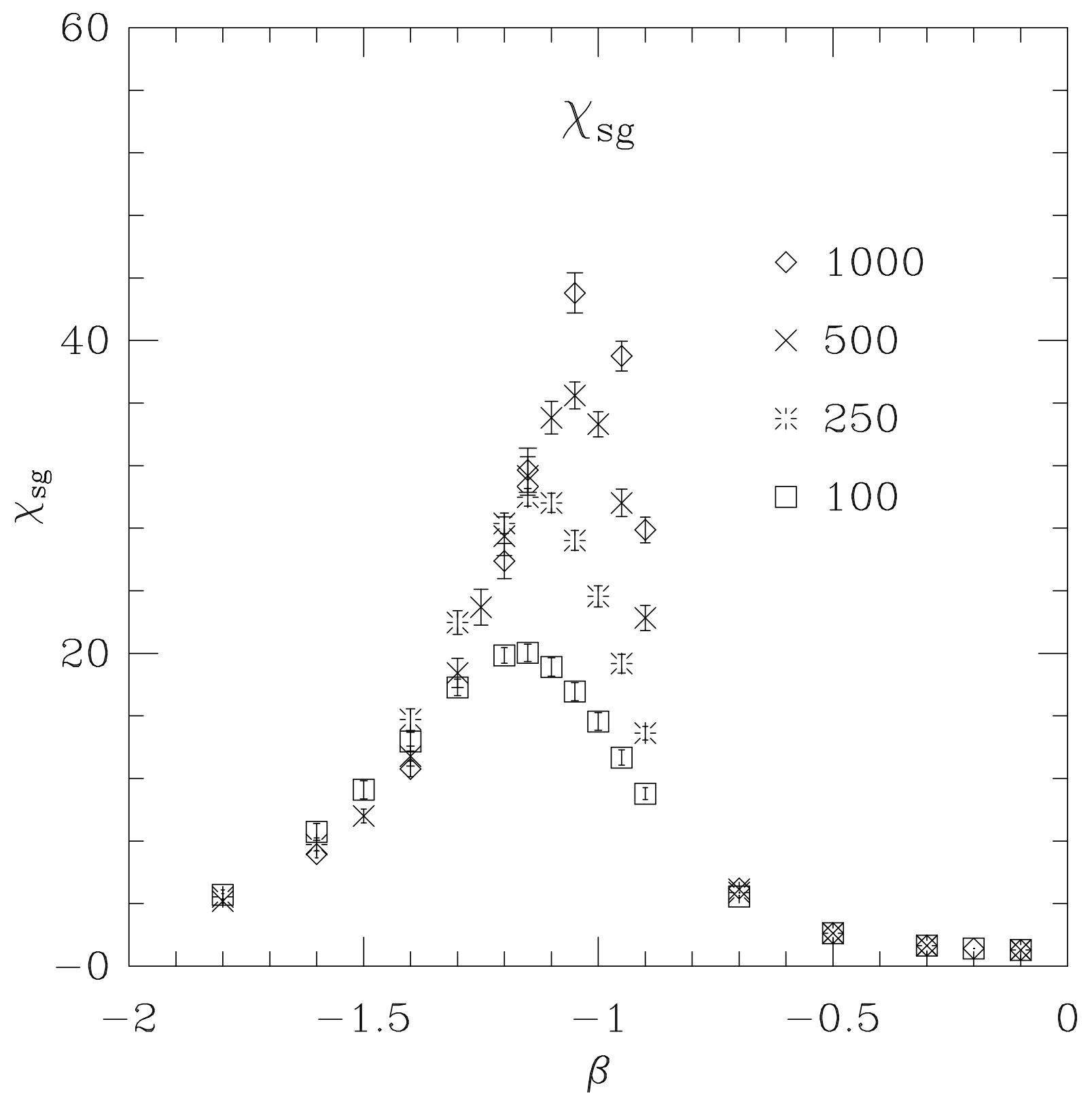


This figure "fig1-4.png" is available in "png" format from: http://arxiv.org/ps/hep-lat/9407024v1 
This figure "fig2-4.png" is available in "png" format from: http://arxiv.org/ps/hep-lat/9407024v1 


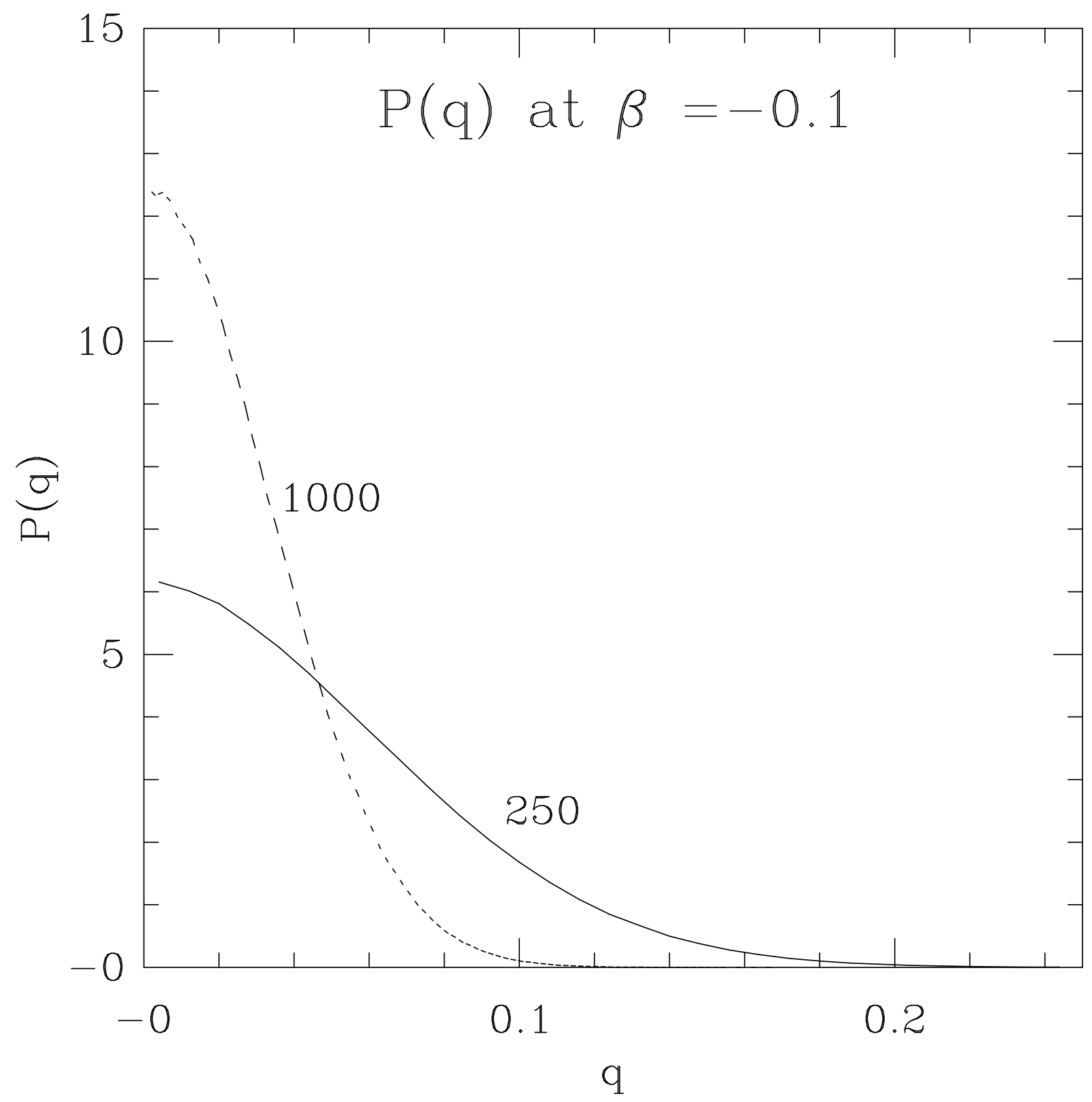


This figure "fig2-5.png" is available in "png" format from: http://arxiv.org/ps/hep-lat/9407024v1 


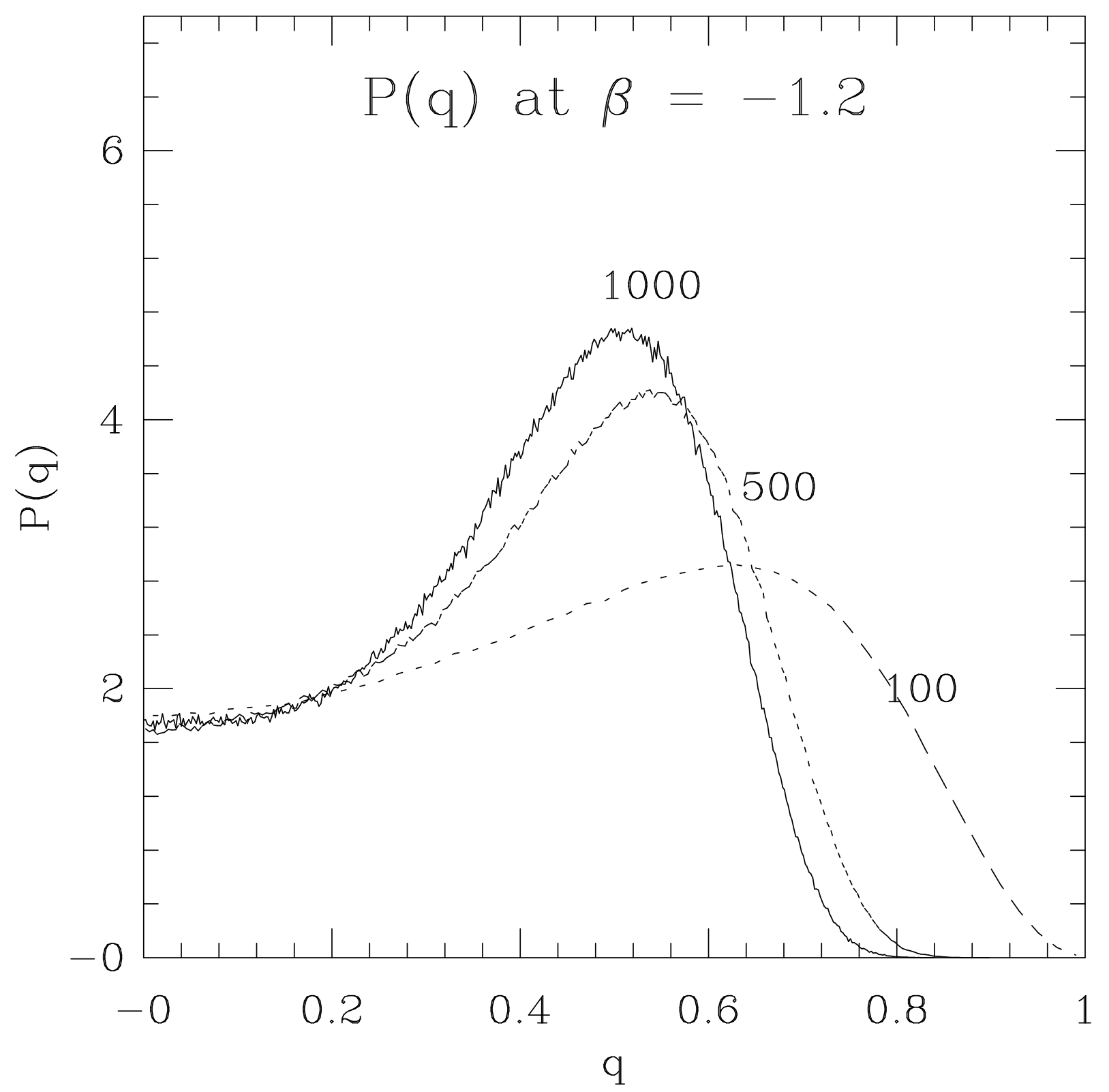

